\title{
Colimits of algebras revisited
}

\section{Jiří Adámek}

It has been open for some time whether, given an algebraic theory (triple, monad) $\pi$ in a cocomplete category $K$, also the category $k^{\pi}$ of $\pi$-algebras must be cocomplete. We solve this in the negative by exhibiting a free algebraic theory $\pi$ in the category Gra of graphs such that $G$ a ${ }^{\Pi}$ is not cocomplete. Further, we improve somewhat the well-known colimit theorem of Barr and Linton by showing that the base category need not be complete.

\section{Categories of algebras...}

I. 1. Is it true that an arbitrary theory of continuous (or ordered or compact, and so on) algebras allows the formation of sums? More generally: given an algebraic theory $\pi$ in a "decent" cocomplete category $K$, is it true that the category $k^{\Pi}$ of $\Pi$-algebras is also cocomplete? While analogous questions about limits are elementary (the forgetful functor $K^{\pi} \rightarrow K$ always creates limits), colimits present an interesting problem. Various sufficient conditions (which cover all of the important cases, in fact) have been found. For example, Linton proved in [7]:

THEOREM (Linton). If $K$ has sums and $K^{\pi}$ has coequalizers then $K^{\Pi T}$ is cocomplete.

I.2. Other conditions involve factorization systems. Let us recall (for example from [5] or [9]) that a factorization system (E, $M$ ) in a category $K$ consists of classes $E, M$ of morphisms subject to the following conditions:

Received 26 July 1977. 
(i) all $M$-morphisms are monos, all E-morphisms are epis;

(ii) $M$ and $E$ are subcategories, that is, closed to composition, both containing all isomorphisms;

(iii) $K=M . E$, that is every morphism $f$ has a factorization $f=m \cdot e$ with $e \in E$ and $m \in M$;

(iv) diagonal fill-in: for every commutative square

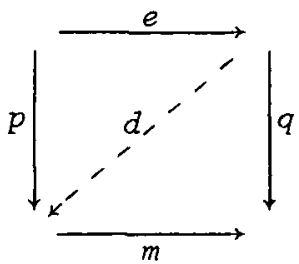

with $e \in E, m \in M$, there exists a (diagonal) morphism $d$, making both triangles commute.

Factorization systems have a lot of natural properties, easy to verify, such as the following:

(v) $E$ contains all coequalizers (this is an exercise in [5]);

(vi) opposite an E-morphism in a pushout there is an E-morphism (see [9]);

(vii) a multiple pushout of $E$-morphisms consists of E-morphisms (this is proved, more generally, in IV.I below).

I.3. The following important theorem has been proved by Linton [7] and, in a different way, by Barr [4].

THEOREM (Barr and Linton). Let $K$ be a category with a factorization system $(E, M)$ which is

(a) complete,

(b) cocomplete,

(c) E-coweZl-powered.

Let $\Pi=(T, \mu, n)$ be an algebraic theory which preserves $E$; that is such that $e \in E$ implies $T e \in E$. Then the category $K^{\pi}$ is cocomplete. 
(Neither Barr nor Linton used the above definition of a factorization system; but we show in Section IV that their definitions are equivalent to ours. Linton supposed that $\pi$ preserves also $M$.)

I.4. Two of the assumptions in the above colimit theorem can be felt as not entirely natural: completeness (cannot we do without it in a colimit theorem?) and preservation of $E$ (is it necessary to assume things not only about $K$ but also about $\Pi$ ?). The aim of the present paper is to show that completeness is redundant (see section II) while preservation of $E$ is not (see section III).

Let us remark that Barr exhibits in [4] another colimit theorem: if $\pi$ has rank then $k^{\pi}$ is cocomplete. This covers all "natural" theories $\pi$. Thus, it is no surprise that the counterexample in Section III consists of an ugly algebraic theorem $\Pi$ (in a nice category $K$, though).

$$
\text { II. ... are often cocomplete... }
$$

II.l. We shall consider not only $\pi$-algebras of an algebraic theory but, more generally, $F$-algebras of an arbitrary endofunctor $F: K \rightarrow K$. An $F$-algebra is a pair $(A, \alpha)$, consisting of an object $A$ of $K$ and a morphism $\alpha: F A \rightarrow A$ (subject to no axioms). Given two $F$-algebras $(A, \alpha)$ and $(B, B)$, by an $F$-homomorphism $f:(A, \alpha) \rightarrow(B, B)$ is meant a K-morphism $f: A \rightarrow B$ such that $f . \alpha=\beta . F f$. We denote by $K(F)$ the category of $F$-algebras and $F$-homomorphisms.

Thus, given an algebraic theory $\pi=(T, \mu, \eta)$ in $K$ the category $K^{\pi}$ of $\pi$-algebras is a full subcategory of the category $K(T)$ of $T$-algebras .

II.2. Categories $K(F)$ were used by Barr [4] for the study of free algebraic theories - this study was then applied by Arbib and Manes [3] to automata in categories. The latter call $F$ an input process provided that the forgetful functor $K(F) \rightarrow K$ has a left adjoint, in other words, provided that each object $A$ in $K$ generates a free $F$-algebra. Explicitly, this free $F$-algebra consists of an $F$-algebra $\left(A^{\#}, \varphi^{A}\right)$ and a morphism $s^{A}: A \rightarrow A^{\#}$ in $K$ which is universal in the following sense. Given an $F$-algebra $(B, B)$, for every morphism $f: A \rightarrow B$ there is a unique F-homomorphism $f^{\#}:\left(A^{\#}, \varphi^{A}\right) \rightarrow(B, B)$ with $f=f^{\#} \cdot s^{A}$. 
For each input process $F$ there arises an algebraic theory $\pi$ (freely generated by $F$ ) with

$$
\begin{aligned}
& T A=A^{\#} ; \\
& \mu^{A}: A^{\# \#} \rightarrow A^{\#} \text { is the unique } F \text {-homomorphism } \\
& \qquad\left(A^{\# \#}, \varphi^{A^{\#}}\right) \rightarrow\left(A^{\#}, \varphi^{A}\right) \text { with } \mu^{A} \cdot s^{A}=1_{A} ; \\
& \eta^{A}=s_{A}: A \rightarrow A^{\#} .
\end{aligned}
$$

Barr [4] proves that, under additional assumptions on $K$, these are the only free algebraic theories in $K$.

PROPOSITION (Barr). Let $F$ be an input process and let $\pi$ be the corresponding free algebraic theory. Then the categories $K(F)$ and $K^{\pi}$ are isomorphic.

II.3. When aiming at a cocompleteness theorem for categories $k^{\pi}$, we can restrict our attention to coequalizers in $K^{\pi}$ (I.I); it turns out that, sufficiently often, we can work with coequalizers in $K(T)$ :

LEMMA. Let $K$ be a category with a factorization system ( $E, M)$, let $\Pi=(T, \mu, \eta)$ be an algebraic theory, preserving $E$. Then for every coequalizer in $K(T)$,

$$
(A, \alpha) \stackrel{f}{\stackrel{f}{\longrightarrow}}(B, \beta) \stackrel{k}{\longrightarrow}(C, \gamma)
$$

such that $(B, \beta)$ is a T-algebra, also $(C, \gamma)$ is a T-algebra.

Proof. Let $E^{T}$ denote the class of all T-homomorphisms with underlying morphism in $E$; analogously $M^{T}$. Then $\left(E^{T}, M^{T}\right)$ is a factorization system in $K(T)$; see [9], 3.4.17. Hence, by I.2 (v), $k \in E$. By hypothesis, also $T k \in E, T^{2} k \in E$, and so on.

To show that $(C, \gamma)$ is indeed a $\Pi$-algebra, consider the following diagrams, which clearly comute: 

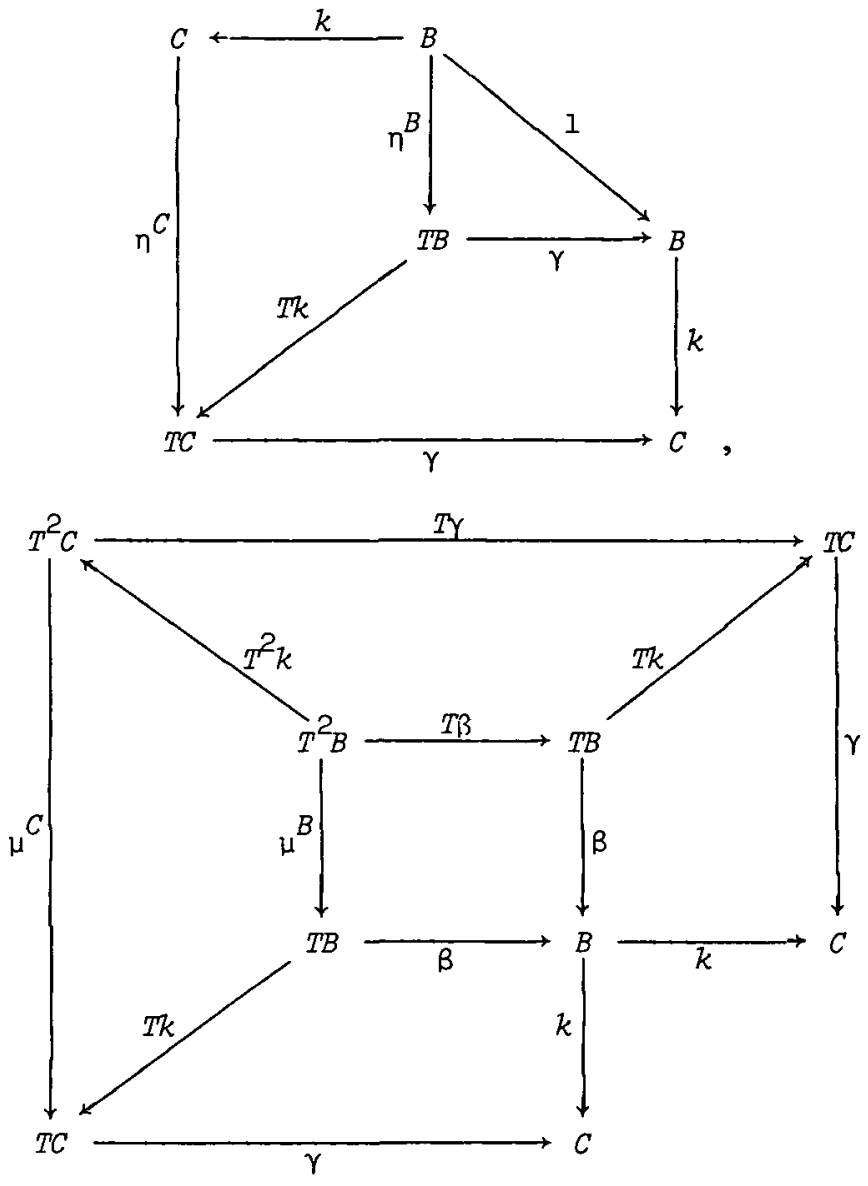

By the first one, $\left(\gamma \cdot \eta^{C}\right) \cdot k=k$, hence $\gamma \cdot n^{C}=1$ ( $k$ is epi). By the second one, $\left(\gamma \cdot \mu^{C}\right) \cdot T^{2} k=(\gamma \cdot T \gamma) \cdot T^{2} k$; hence $\gamma \cdot \mu^{C}=\gamma T \gamma\left(T^{2} k\right.$ is epi).

II.4. The following theorem is proved in [2] in a different manner, as a part of a more general study of colimits in $K(F)$. (An iterative colimit-construction is exhibited there, generalizing that used in universal algebra.) We present a straightforward proof. The help of Václav Koubek with this proof is gratefully acknowledged.

THEOREM. Let $K$ be a cocomplete category with a factorization system $(E, M)$; let $K$ be E-cowell-powered. Then for every functor $F: K \rightarrow K$ which preserves $E$, the category $K(F)$ has coequalizers.

Proof. Let $f, g:(A, \alpha) \rightarrow(B, B)$ be arbitrary $F$-homomorphisms . 
Denote by $\Omega$ the class of all $E$-epis $t: B \rightarrow T$ in $K$ with the following property :

for every $F$-homomorphism $h:(B, B) \rightarrow(C, \gamma)$ with $h . f=h . g$ there exists $h_{(t)}: T \rightarrow C$ in $K$ such that $h=h_{(t)} \cdot t$.

Since $K$ is cocomplete and $E$-cowell-powered, the diagram $\Omega$ has a colimit (multiple pushout)

$$
r_{0}=r_{(t)} \cdot t: B \rightarrow T_{0}\left(r_{(t)}: T \rightarrow T_{0} \text { for each } t \in \Omega\right) \text {. }
$$

Each $t \in \Omega$ is in $E$, hence (by $I .2(v i i)$ ) each $r_{(t)}$ is in $E$; thus $t_{0} \in E$ and $F t_{0} \in E$.

Fix a homomorphism $h:(B, \beta) \rightarrow(C, \gamma)$ with $h \cdot f=h . g$. Then we have a bound of $\Omega: h_{(t)}: T+C \quad(t \in \Omega)$. Thus there exists

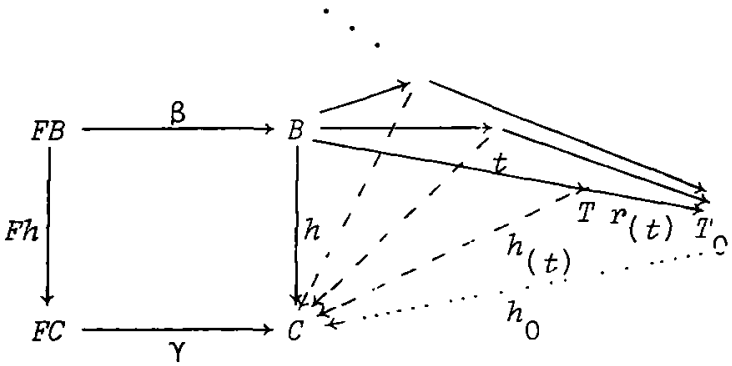

a unique $h_{0}: T_{0} \rightarrow C$ with

$$
h_{0} \cdot r_{(t)}=h_{(t)} \quad(t \in \Omega) \text { and } h_{0} \cdot t_{0}=h .
$$

Consider the pushout of $F t_{0}$ and $t_{0} \cdot B$ :

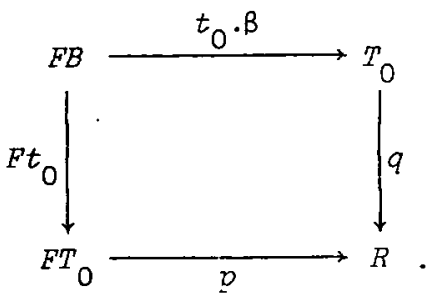

CLAIM. $q$ is an isomorphism. It suffices to show that $q \cdot t_{0} \in \Omega$; then by (1), $t_{0}=r\left(q \cdot t_{0}\right) \cdot q \cdot t_{0}$, which implies $1=r\left(q \cdot t_{0}\right) \cdot q$ since $t_{0}$ 
is epi) and so $q$ is a split mono as well as an E-epi (opposite $F t_{0} \in E$ in a pushout, see I.2 (vi)) - thus, $q$ is an isomorphism. To show $q \cdot t_{0} \in \Omega$ we first remark that, since $q \in E$ and $t_{0} \in E$ we have $q \cdot t_{0} \in E$. Secondly, consider any homomorphism $h:(B, \beta) \rightarrow(C, \gamma)$ with $h \cdot f=h \cdot g:$ we have $h_{0} \cdot t_{0}=h$ by (2) and $h \cdot \beta=\gamma \cdot F h$, hence

$$
h_{0} \cdot\left(t_{0} \cdot \beta\right)=\gamma \cdot F h=\left(\gamma \cdot F h_{0}\right) \cdot F t_{0} \cdot
$$

This implies that the pair $h_{0} ;\left(\gamma \cdot F h_{0}\right)$ factorizes through $q ; p$ above; that is, there is a unique morphism, denoted by $h\left(q \cdot t_{0}\right)$ from $R$ to $C$,

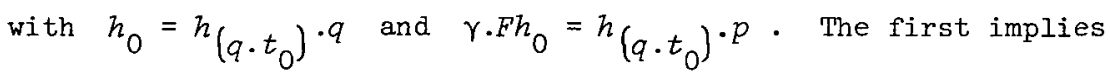

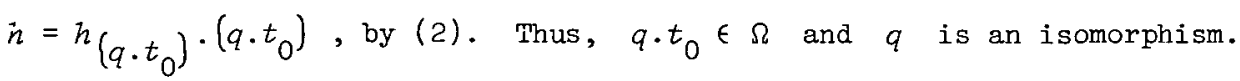
Let us show that the $F$-homomorphism $t_{0}:(B, \beta) \rightarrow\left(T_{0}, q^{-1} \cdot p\right)$ is a coequalizer of $f$ and $g$ in $K(F)$.

Firstly, $t_{0} \cdot f=t_{0} \cdot g$ : indeed, consider the coequalizer $c$ of $f$ and $g$ in $K ; c \in E$ by I.2 (v), and clearly $c \in \Omega$. Hence $t_{0}=r_{(c)} \cdot c$, which proves $t_{0} \cdot f=t_{0} \cdot g$.

Secondly, for every homomorphism $h:(B, \beta) \rightarrow(C, \gamma)$ with $h \cdot f=h \cdot g$ we have $h_{0}: T_{0} \rightarrow C$ with $h=h_{0} \cdot t_{0}$, by (2). This $h_{0}$ is unique, because $t_{0}$ is epi. To conclude the proof we only have to show that $h_{0}$ is a homomorphism; that is, that $h_{0} \cdot\left(q^{-1} \cdot p\right)=\gamma \cdot F h_{0} \cdot$ We use (2) and the fact that $F t_{0} \in E$ is epi, and that $p \cdot F t_{0}=q \cdot t_{0} \cdot \beta$ (see the pushout above):

$$
\begin{aligned}
{\left[h_{0} \cdot\left(q^{-1} \cdot p\right)\right] \cdot F t_{0} } & =h_{0} \cdot\left(q^{-1} \cdot q\right) \cdot t_{0} \cdot \beta \\
& =h \cdot \beta \\
& =\gamma \cdot F h^{-\beta} \\
& =\left[\gamma \cdot F h_{0}\right] \cdot F t_{0} .
\end{aligned}
$$

II.5. COROLLARY. Let $K$ and $(E, M)$ be as in II.4. Then for every 
algebraic theory $\pi$ which preserves $E$, the category $k^{\pi}$ is cocomplete.

Proof. By II.4, the category $K(T)$ has coequalizers, hence (by II.3) so does $K^{\pi}$. By I.I this implies the cocompleteness.

COROLLARY. Let $K$ be a cowezz-powered, cocomplete category. Then for every algebraic theory $\pi$ preserving epis, also $K^{\Pi}$ is cocomplete.

Proof. It is proved in [5] (the dual to 34.1) that $K$ has a factorization system $(E, M)$ with $E$ equal to all epis, $M$ equal to all extremal monos.

II.6. The latter corollary is proved in [1] in the same way as in the present paper. The first corollary was first formulated by Reiterman. See [6], where a completely different method is used (related to that used in [2] to prove Theorem II. 4 above).

\section{III. ... but not always!}

III.]. We denote by Gra the category of graphs and compatible mappings. A graph is a pair $A=\langle A, K\rangle$ consisting of a set $A$ and a subset $K$ of $A \times A$. A compatible mapping $f:\langle A, K\rangle \rightarrow\langle B, L\rangle$ is a mapping $f: A \rightarrow B$ for which $(x, y) \in K$ implies $(f(x), f(y)) \in L$.

Gra is a complete and cocomplete concrete cutegory, with underlying functor Gra $\rightarrow$ Set creating all limits and colimits; it is also a wellpowered and cowell-powered category and is, in one word, decent.

III.2. We shall define an input process $F$ in Gra such that the category $G r a(F)$ of $F$-algebras is not cocomplete. Before doing this, we shall make a simple observation about $P$-algebras, where $P$ : Set $\rightarrow$ Set is the power-set functor (sending a set $X$ to the power set $P X=2^{X}$ and a mapping $f: X \rightarrow Y$ to the mapping

$$
\text { Pf : } A \mapsto\{f(a) ; a \in A\}) \text {. }
$$

We recall that an object $O$ of a category is weak initial if for every other object $X$ there exists at least one morphism from $O$ to $X$.

LEMMA. The category Set $(P)$ of P-algebras has no weak initial object.

Proof. It is easy to see that $\operatorname{Set}(P)$ is a complete category (with 
limits created by the forgetful functor $\operatorname{Set}(P) \rightarrow$ Set $)$. Thus the existence of a weak initial object would imply the existence of an initial object; see [8].

Now let $(A, \alpha)$ be an initial $P$-algebra. Barr proves in [4] that $\alpha$ is then an isomorphism. But there exists no isomorphism from a power set $P A$ to $A$, of course; a contradiction.

III.3. We start by defining a functor $F:$ Gra $\rightarrow$ Gra . First, for every graph $\mathrm{A}=\langle A, K\rangle$, define a set

$$
A^{(3)}=\{(x, y, z) \in A \times A \times A ;(x, y) \in K \text { and }(y, z) \in K\} \text {. }
$$

Given a compatible mapping $f: A \rightarrow B$, define a mapping $f^{(3)}: A^{(3)} \rightarrow B^{(3)}$ by

$$
f^{(3)}:(x, y, z) \mapsto(f(x), f(y), f(z)) .
$$

Now define $F$ as follows: for each graph $A$ put

$F A=\left\langle P A^{(3)}, M_{A}\right\rangle$ where $(X, Y) \in M$ iff $X=\emptyset$ and $Y \neq \varnothing\left(X, Y \subset A^{(3)}\right)$; for each compatible map $f: A \rightarrow B$ put

$$
F f=P f^{(3)} \text {. }
$$

Clearly, $P f^{(3)}: F A \rightarrow F B$ is compatible and $F$ is a correctly defined functor.

III.4. LEMMA. $F$ is an input process.

Proof. For every graph A define a new graph

$$
A^{\#}=A \vee F A
$$

and notice that $(E A)^{(3)}=\emptyset$; hence $F A^{\#}=F A$. Denote by $s^{A}: A \rightarrow A^{\#}$, $\varphi^{A}: F A^{\#}=F A \rightarrow A^{\#}$ the canonical injections. Then $\left(A^{\#}, \varphi^{A}\right)$ is a free $F$-algebra generated by $A$ with universal morphism $s^{A}$.

Indeed, let $(B, B)$ be an $F$-algebra and let $f: A \rightarrow B$ be a morphism (that is a compatible mapping). Define $f^{\#}: A^{\#} \rightarrow B$ by

$$
f^{\#} \cdot \varphi^{A}=B \cdot F f \text {, }
$$




$$
f^{\#} \cdot s^{A}=f \text {. }
$$

Since $(F A)^{(3)}=\emptyset$, clearly $F f^{\# \#}=F f$, and so (3) means that $f^{\#}:\left(A^{\#}, \varphi^{A}\right) \rightarrow(B, B)$ is an $E$-homomorphism; by (4), $f^{\#}$ extends $f$. The uniqueness of $f^{\#}$ follows from the fact that (3) and (4) are actually necessary .

III.5. We define a pair $f, g:(A, \alpha) \rightarrow(B, B)$ of $F$-homomorphisms of which we shall prove that they do not have a coequalizer in Gra( $F)$.

Let $A=\{\{p, q\}, \emptyset)$ and $B=\{\{s, t\},\{(s, t)\})$.

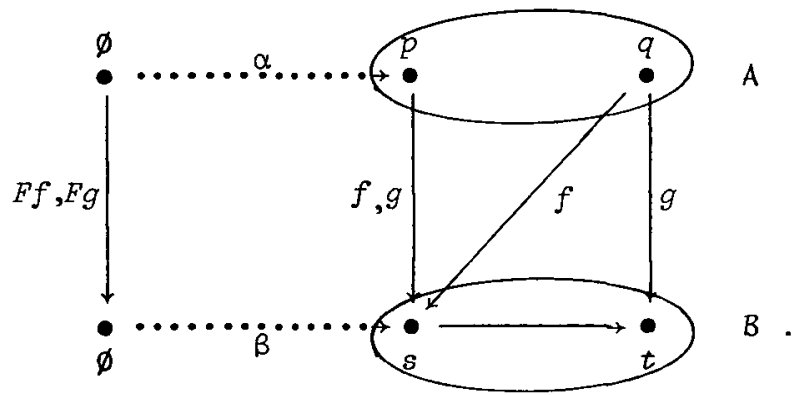

Clearly $A^{(3)}=B^{(3)}=\emptyset$, hence $F A=F B=\langle\{\emptyset\}, \emptyset\rangle$. Define $\alpha: F A \rightarrow A$ by $\alpha(\emptyset)=p ; \beta: F B \rightarrow B$ by $B(\varnothing)=s$.

Finally, define $f, g:\{p, q\} \rightarrow\{s, t\}$ by

$$
f(p)=g(p)=f(q)=s \text { and } g(q)=t \text {. }
$$

Clearly, $f$ is a homomorphism with $f \cdot \alpha=\beta . F f: \emptyset \vdash s$, analogously $g$.

III.6. Assuming that $f, g$ have a coequalizer $c:(B, B) \rightarrow(C, \gamma)$ in $G r a(F)$, we shall find a weak initial object in $\operatorname{Set}(P)$ - a contradiction.

We have $c=(C, K)$. Put $\bar{s}=c(s)(=c(t)$, because $c \cdot f=c \cdot g)$. Since $c: B+C$ is compatible, clearly $(\bar{s}, \bar{s}) \in K$.

Put

$$
C_{0}=\{x \in C ;(\bar{s}, x) \in K\} \text {. }
$$

For every subset $X \subset C_{0}$ put

$$
\hat{X}=\{(\bar{s}, \bar{s}, x) ; x \in X\} \in P C^{(3)} \text {. }
$$


We have $\gamma(\hat{X}) \in C$ - let us show that, in fact, $\gamma(\hat{X}) \in C_{0}$. If $X=\emptyset$, then $\hat{X}=\emptyset$ and $\gamma(\emptyset)_{-}=\bar{s} \in C_{0}$, because $\gamma \cdot F c=c \cdot \beta$ and $F c(\phi)=\emptyset$; thus

$$
\gamma(\emptyset)=c(\beta(\varnothing))=c(s)=\bar{s} .
$$

If $X \neq \varnothing$, then $\hat{X} \neq \varnothing$ and so $(\varnothing, \hat{X}) \in M_{C} \quad$ (see III.3). Since $\gamma: F C \rightarrow C$ is compatible, this yields $(\gamma(\varnothing), \gamma(\hat{X})) \in K$; that is,

$$
\{s, \gamma(\hat{X})) \in K \text {; thus } \gamma(\hat{X}) \in C_{0} \text {. }
$$

Now we define a P-algebra $\left(G_{0}, \hat{\gamma}\right)$ by

$$
\hat{\gamma}(X)=\gamma(\hat{X}) \quad\left(X \subset c_{0}\right) \text {. }
$$

This $P$-algebra is weak initial.

Proof. Let $(M, \mu)$ be another $P$-algebra, that is, a set $M$ and a mapping $\mu: P M \rightarrow M$; put $m_{0}=\mu(\emptyset)$. Define an $F$-algebra $\left(M, \mu^{*}\right): M=\left(M,\left\{\left(m_{0}, m\right) ; m \in M\right\}\right)$ where $\mu^{*}: F M \rightarrow M$ is defined by

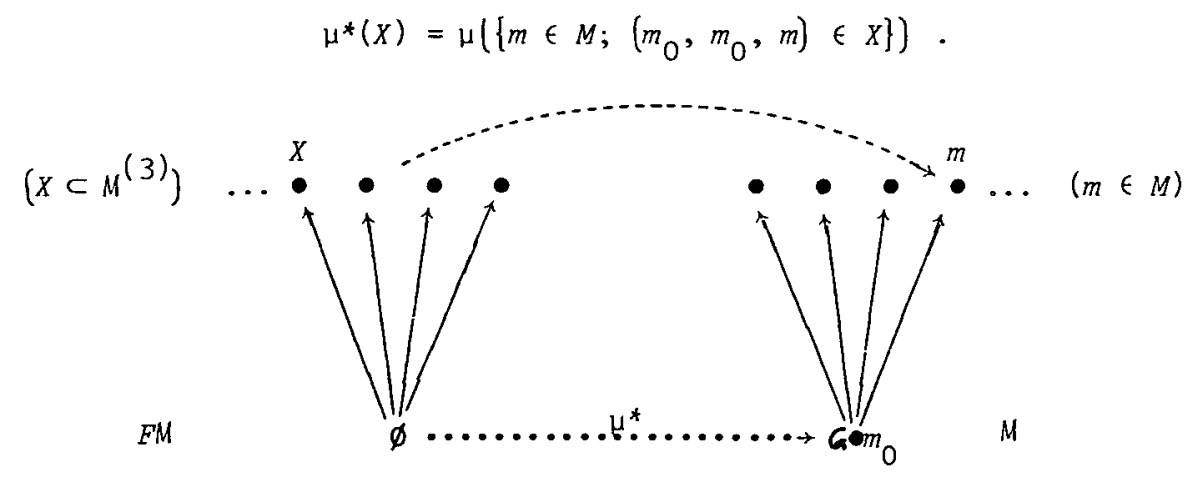

Particularly, $\mu^{*}(\varnothing)=m_{0}$. Thus $h:(B, B)+\left(M, \mu^{*}\right)$, defined by $h(s)=h(t)=m_{0}$, is an $E$-homomorphism. Since $h \cdot f=h \cdot g$, there exists an $E$-homomorphism $k:(C, \gamma) \rightarrow\left(M, \mu^{*}\right)$ such that $k . c=h-$ particularly, $k(\bar{s})=m_{0}$.

The proof will be concluded when we show that the restriction $k_{0}: C_{0} \rightarrow M$ of $k$ is a $P$-homomorphism; that is, that $k_{0} \cdot \hat{\gamma}=\mu . P k_{0}$. Given $X \subset C_{0}$ we have 


$$
k_{0} \cdot \hat{\gamma}(X)=k \cdot \gamma(\hat{X})=\mu^{*} \cdot F k(\hat{X})
$$

Furthermore, $\hat{X}=\{(\bar{s}, \bar{s}, x) ; x \in X\}$ implies

$$
F k(\hat{X})=\left\{\left(m_{0}, m_{0}, k(x)\right) ; x \in X\right\}
$$

and $k(x)=k_{0}(x)$ for $x \in X$ (since $\left.X \subset c_{0}\right)$; thus

$$
\mu^{*} \cdot F k(\hat{X})=\mu\left(\left\{k_{0}(x) ; x \in X\right\}\right)=\mu \cdot P k_{0}(X) .
$$

Thus $\left(C_{0}, \hat{\gamma}\right)$ is a weak initial $P$-algebra, in contradiction to Lema III. 2 .

CONCLUSION. The free algebraic theory $\pi$ generated by the above input process $F$ in Gra is such that $G r a{ }^{\Pi}$ is not complete. Explicitly, $\pi=(T, \mu, n)$ with

$$
T A=A \vee F A \quad\left(n^{A}: A \rightarrow T A \text { and } \varphi^{A}: F A \rightarrow T A \text { canonical }\right)
$$

and $\mu^{A}: T^{2} A=(A \vee F A) \vee F A \rightarrow T A$ is defined on $A$ as $\eta^{A}$ and on both copies of $F A$ as $\varphi^{A}$.

\section{Appendix on factorizations}

IV.1. Barr's right factorization systems. For the colimit theorem of I.3, Barr [4] uses a right factorization system, which is a pair (E, $M)$ as in I.2, except that $E$-morphisms need not be epis. More precisely, a right factorization system consists of a class $E$ of morphisms and a class $M$ of monos such that conditions (ii)-(iv) of I.2 are fulfilled.

There always exists a simple right factorization system: $E$ equals all morphisms, $M$ equals all isomorphisms. In this case, $K$ is seldom E-cowell-powered (as required in the colimit theorem I.3). We shall show that this is no coincidence.

LEMMA. For each right factorization system $(E, M)$ and each multiple pushout 


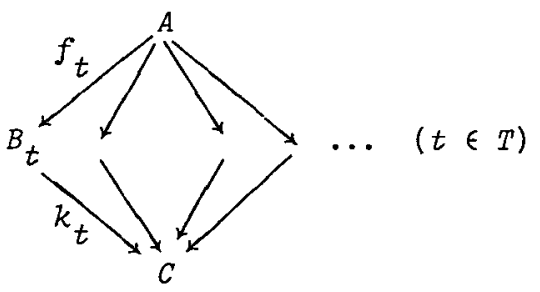

with $f_{t} \in E$ for $t \in T$, also $k_{t} \in E$ for $t \in T$.

Proof. Choose $t_{0} \in T$ and let $k_{t_{0}}=m . e$ be an $E-M$-factorization. For every $t \in T$ use the diagonal fill-in:

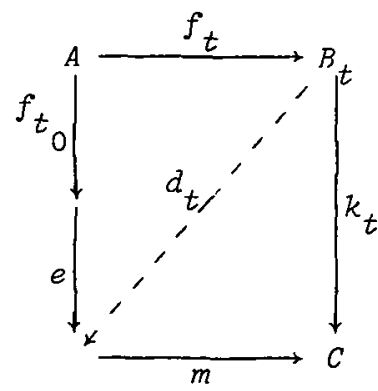

to obtain $d_{t}$ with $d_{t} \cdot f_{t}=e \cdot f_{t_{0}}$ (hence $d_{t}$ is a bound of the pushout) and $m \cdot d_{t}=k_{t}$. There exists a unique $d$ with $d_{t}=d \cdot k_{t}(t \in T)$.

Then $(m . d) . k_{t}=k_{t}(t \in T)$; hence $m \cdot d=1$. Since $m \in M, m$ is a mono as well as a split epi - thus $m$ is an isomorphism. This shows that $k_{t_{0}}=m \cdot e$ is in $E$.

PROPOSITION. Every right factorization system (E, M) in a cocomplete, E-cowe IL-powered category is a factorization system (that is, all E-morphisms are epis).

Proof. Assume that $K$ is a cocomplete category with a right factorization system $(E, M)$. Given $f: A \rightarrow B$ in $E$ which is not epi, we shall show that $K$ is not $E$-cowell-powered. Indeed, if $K$ is E-cowell-powered, there exist $q_{i}: B \rightarrow Q_{i}(i \in I, I$ a set $)$ in $E$ such that each E-morphism with domain $B$ is isomorphic to some $q_{i}$. Choose a cardinal $\lambda$ such that card hom $\left(B, Q_{i}\right)<\lambda$ for each $i \in I$. 
Let $\left\{k_{t}\right\}_{t \in T}$ be the multiple

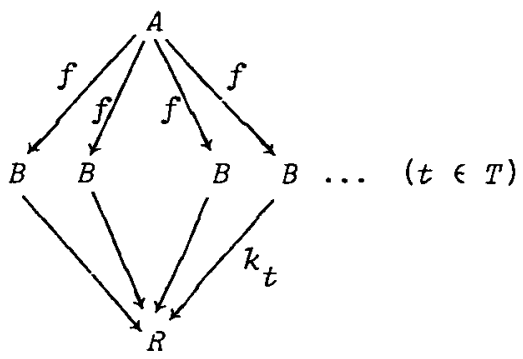

pushout of a $T$-indexed family of copies of $f$, where $T$ is a set of power $\lambda$. By the above lemma, $k_{t} \in E$ for each $t \in T$. To conclude the proof it suffices to show that the $k_{t}$ 's are pairwise distinct: then card $\operatorname{hom}(B, R) \geq \lambda$; hence $R$ is not isomorphic to any of $Q_{i}$.

Since $f$ is not epi, there exist distinct morphisms $g_{1}, g_{2}: B \rightarrow C$ with $g_{1} \cdot f=g_{2} \cdot f$. Consider the following bound $g_{n_{t}}: B \rightarrow C$ of the above pushout: for a given $t_{0} \in T, n_{t_{0}}=1$; else $n_{t}=2$. There exists a unique $h: R \rightarrow C$ with $g_{n_{t}}=h \cdot k_{t}(t \in T)$. Since $g_{n_{t_{0}}} \neq g_{n_{t}}$, we have $k_{t_{0}} \neq k_{t}$ for each $t_{0} \neq t$. This shows that the $k_{t}$ 's are pairwise distinct.

IV.2. Linton's factorization functors. Denote by $k^{2}$ the morphism category of $k$ and by $k^{3}$ the triangle category of $k$ (objects are triples $(f ; g, h)$ of $K$-morphisms with $f=g . h ;$ morphisms are triples $(p, r, q):(f ; g, h) \rightarrow\left(f^{\prime} ; g^{\prime}, h^{\prime}\right)$ of K-morphisms with $r \cdot h=h^{\prime} \cdot p$ and $\left.q \cdot g=g^{\prime} \cdot q\right)$. There is a natural forgetful functor $\gamma: k^{3}+k^{2}$ (sending $(f ; g, h)$ to $f)$.

Linton [7] uses a factorization functor, that is a functor

$$
\Delta: k^{2} \rightarrow k^{3}
$$

such that 
(1) $\gamma \circ \Delta=1$; for $f: X \rightarrow Y$ in $k^{2}, \Delta(f)$ is denoted by

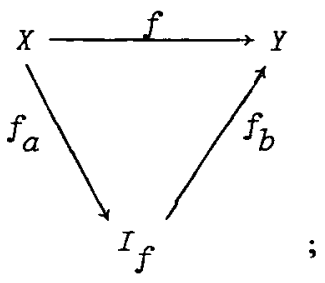

(2) $f_{a}$ is epi, $f_{b}$ is mono, for each $f \in k^{2}$;

(3) $\left(f_{a}\right)_{b}$ and $\left(f_{b}\right)_{a}$ are isomorphisms, for each $f \in K^{2}$.

PROPOSITION. (A) Given a factorization functor $\Delta$, the pair $\left(E_{\Delta}, M_{\Delta}\right) \cdot$ with

$$
\begin{aligned}
& E_{\Delta}=\left\{f \in K^{2} ; f_{b} \text { is an isomorphism }\right\}, \\
& M_{\Delta}=\left\{f \in K^{2} ; f_{a} \text { is an isomorphism }\right\},
\end{aligned}
$$

is a factorization system in $K$.

(B) If $\Delta, \Delta^{\prime}$ are naturally equivalent factorization functors then $E_{\Delta}=E_{\Delta^{\prime}}$, and $M_{\Delta}=M_{\Delta^{\prime}}$.

(C) For every factorization system (E, M) there exists a factorization functor $\Delta$, unique up to natural equivalence, with $(E, M)=\left(E_{\Delta}, M_{\Delta}\right)$.

Proof. (A). Conditions (i), (iii) in I.2 are evident. Condition (iv) follows from the fact that $(p, q): e \rightarrow m$ is a morphism in $k^{2}$; hence we have $\Delta(p, q): \Delta(e) \rightarrow \Delta(m)$ in $k^{3}$. 


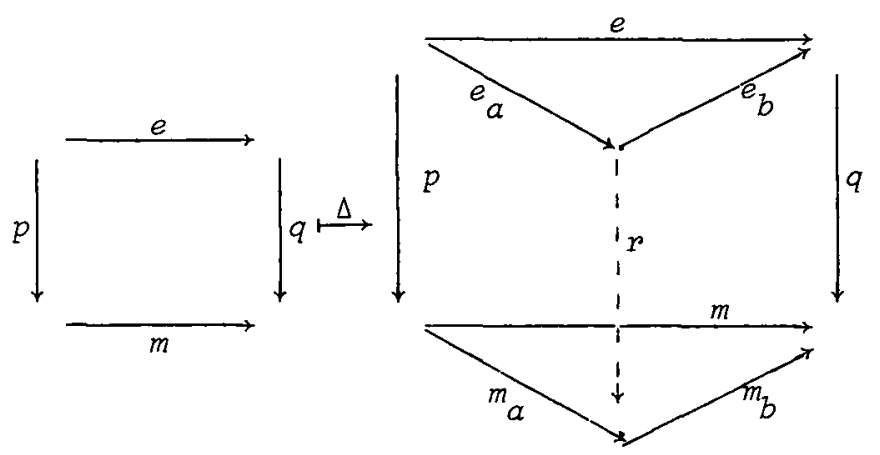

Since $\gamma \circ \Delta=1$, clearly $\Delta(p, q)=(p, r, q)$ for some morphism $r: I_{e} \rightarrow I_{m}$, making the above diagram commute. The diagonal morphism is then

$$
d=m_{a}^{-1} \cdot r \cdot e_{b}^{-1}
$$

Finally, to verify condition (ii) it suffices to show that $E$ and $M$ are closed to composition with isomorphisms; see [5], 33.3. Let us verify, for example, that $e \in E$ implies $e \circ i \in E$ whenever $i$ is an

isomorphism; the rest is analogous. Consider $\left(i^{-1}, 1\right): e \rightarrow e . i$ in $k^{2}$; we have $\Delta\left(i^{-1}, 1\right)$ of the form $\left(i^{-1}, r, 1\right): \Delta(e) \rightarrow \Delta(e \circ i)$ in $k^{3}:$

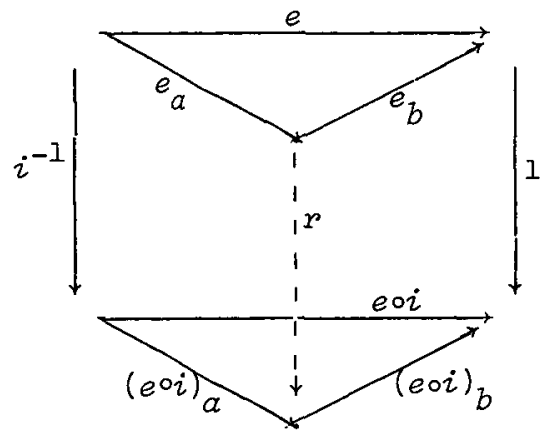

Since $(e \circ i)_{b} \circ r \circ e_{b}^{-1}=1$, we see that $(e \circ i)_{b}$ is a split epi as well as a mono by (2) above. Hence $(e \circ i)_{b}$ is an isomorphism; thus $e \circ i \in E$.

(B) follows easily from the fact that both $E$ and $M$ are closed to 
composition with isomorphisms .

(C). For every morphism $f$ choose a fixed factorization $f=f_{b} \cdot f_{a}$ with $f_{a} \in E, f_{b} \in M$. Put $\Delta(f)=\left(f ; f_{b}, f_{a}\right)$. Given a morphism $(p, q): f \rightarrow g$ in $k^{2}$,

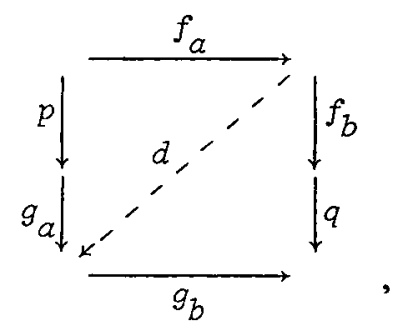

use the diagonal fill-in on the square above to find $d$ and define

$$
\Delta(p, q)=(p, d, q): \Delta(f) \rightarrow \Delta(g) .
$$

This gives rise to a factorization functor $\Delta: k^{2} \rightarrow k^{3}$ with $E=E_{\Delta}$ and $M=M_{\Delta}$. Uniqueness follows from $(B)$.

\section{References}

[1] Jiří Adámek, "Kategoriální teorie automatů a universální algebra" [Categorical theory of automata and universal algebra] (Doctoral Dissertation, Charles University, Prague, 1974).

[2] J. Adámek, V. Koubek, "Algebras and automata over a functor", Kibernetika (to appear).

[3] Michael A. Arbib and Ernest G. Manes, "Machines in a category: an expository introduction", SIAM Rev. 16 (1974), 163-192.

[4] Michael Barr, "Coequalizers and free triples", Math. 2. 116 (1970), 307-322.

[5] Horst Herrlich, George E. Strecker, Category theory: an introduction (Allyn and Bacon, Boston, Massachusetts, 1973).

[6] Václav Koubek, Jan Reiterman, "Categorical constructions of free algebras, colimits and completions of partial algebras", submitted. 
[7] F.E.J. Linton, "Coequalizers in categories of algebras", Seminar on triples and categorical homology theory, 75-90 (Lecture Notes in Mathematics, 80. Springer-Verlag, Berlin, Heidelberg, New York, 1969).

[8] S. Mac Lane, Categories for the working mathematician (Graduate Texts in Mathematics, 5. Springer-Verlag, New York, Heidelberg, Berlin, 1971).

[9] Ernest G. Manes, Algebraic theories (Graduate Texts in Mathematics, 26. Springer-Verlag, New York, Heidelberg, Berlin, 1976).

Faculty of Electrical Engineering, Ceské Vysoké Učeni Technické v Praze, Czechoslovakia. 